\title{
Stephen Friend
}

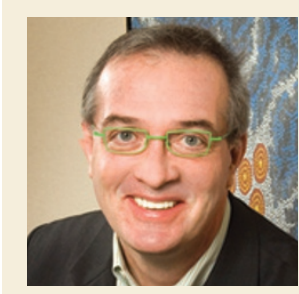

Senior Vice-President and Franchise Head, Oncology, Merck and Company Inc. Stephen Friend co-founded Rosetta Inpharmatics with Leland Hartwell and Leroy Hood in 1996 while at the Fred Hutchinson Cancer Research Center, Seattle, USA. Following the acquisition of Rosetta by Merck in 2001, Friend was instrumental in integrating Rosetta's molecular profiling expertise into research programmes at Merck. In September 2002, he assumed scientific leadership of oncology research at Merck.

What led to the creation of Rosetta Inpharmatics?

Rosetta Inpharmatics grew out of an incubator at the Fred Hutchinson Cancer Center, funded by the government through the National Institutes of Health and companies such as Merck and Chiron. At the time that Rosetta was founded, drug discovery primarily aimed to address very specific questions without taking into account systems approaches - patterns of information about RNA and proteins from human and animal cells. Rosetta established a compendium of known patterns that could inform the effect of unknown compounds or clinical states.

\section{What influence has molecular profiling had} on Merck's drug development?

Since Merck acquired Rosetta 6 years ago, the impact has been at two levels. First, on a technology-platform level, Rosetta provided a robust method for molecular or expression profiling that has impacted Merck's identification of drug development targets and enabled Merck to look at both on- and off-target effects of compounds. The technology has also allowed Merck to identify subpopulations of patients for clinical trials that may allow the development of a differentiated targeted therapy.

The second level of impact is that Rosetta gave Merck an advantage in systems biology. Rosetta started out working on expression profiling but soon progressed to RNA interference (RNAi), microRNAs and the potential to use RNAi as a therapeutic tool. So, Rosetta was a technology platform but it was also a philosophy, an attitude, and an investment in how to develop drugs.

\section{How do you see molecular profiling being used in the future?}

One of the complexities of using small interfering RNA (siRNA) as a therapeutic modality is that you want to optimize on-target and minimize off-target effects. Coupling the use of expression profiling to siRNA as a therapy in a model system allows a simultaneous view of what you expect to see alongside unintended activities. This gives the peripheral vision needed to fine tune the siRNA therapeutic.

We think that expression profiling could help pharma companies identify true signals of efficacy and increase the success rate of drugs going from proof of concept through to Phase III trials. Identifying a subpopulation of patients that are going to respond from day one increases the likelihood that the patient will get value from the drug; it makes the response rates higher and helps use resources more effectively.

The other tool that we have found very effective, which complements expression profiling, is whole-genome RNAi screens as a way to map the pathways that are underlying disease. These screens allow you to knockout, one by one, every target across the genome, almost as if you had the ultimate compound library. So, for a given therapeutic candidate you can find out what is really controlling that pathway. In oncology that's particularly important because we are interested in finding out what combination of drugs will be effective in which patients. The aim is to reduce the current rate of attrition in clinical trials that result from almost random combinations of logical candidates.

What factors have influenced Merck's decision to become more actively involved in the development of anticancer therapies? Four years ago there was a minimal presence of Merck in oncology. We looked at how other companies were doing oncology development and felt that the emphasis on selecting targets was primarily a traditional literature-based approach. We thought that by applying Rosetta-type technology platforms to identify pathways and targets it would give us an advantage for selecting compounds for development and, more importantly, identify patients who would benefit from these therapies.

\section{How has Merck approached building an anticancer drug pipeline?}

In terms of building the anticancer drug pipeline we have nine candidates that are now in publicly disclosed development, four of which have come from an active strategy of in-licensing. We used our profiling tools to validate targets and in-licensed anything that achieved a high validation. The rest have come from internal programmes that took advantage of the knowledge we had about targets from our technology platforms.

What do you think are the main challenges to the co-development of novel anticancer therapies and companion diagnostics, and how is Merck addressing these? One of the main challenges is acknowledging the complexity of cancer. We have begun to see the transition from cytotoxic and hormonal therapies to targeted therapies. I think that over the next 5 years, the leaders in oncology are likely to position their new drugs for specific subpopulations defined by tumour types and molecular markers. If we build drugs that take into account that complexity, then in the next 5-10 years we should see significant increases in the response rates.

Although we are excited about the potential of RNAi therapeutics, delivery remains a major challenge. We are very eager to identify cutting edge advances in delivery to allow the application of the power of RNAi technology.

Finally, a huge challenge will be dealing with non-scientific issues for collecting the necessary information and ensuring anonymity across the cultures of government, regulators, universities and industry. I don't think that industry, government or academia alone will be able to deliver what we need to the patients. Only collaboration will build the databases that will allow the best patient care. 